\title{
Estimativas da produção e propriedades da madeira de eucalipto em Sistemas Agroflorestais
}

\author{
Estimates of production and properties of \\ eucalyptus wood in Agroforestry Systems
}

\author{
Carlos Moreira Miquelino Eleto Torres ${ }^{1}$, Aylson Costa Oliveira ${ }^{2}$, \\ Bárbara Luísa Corradi Pereira ${ }^{2}$, Laércio Antônio Gonçalves Jacovine ${ }^{3}$, \\ Silvio Nolasco de Oliveira Neto ${ }^{4}$, Angélica de Cássia Oliveira Carneiro ${ }^{4}$ e \\ Caio Moreira Miquelino Eleto Torres ${ }^{5}$
}

\begin{abstract}
Resumo
A madeira de eucalipto proveniente de Sistemas Agroflorestais (SAF's) pode ser usada para diversos fins, como lenha, moirões, madeira serrada e carvão vegetal. Quando utilizada para fins energéticos, sobretudo na forma de lenha, é obtida, principalmente, pelo desbaste e apresenta menores diâmetros. Objetivo-se com este trabalho estimar a produção volumétrica e determinar as propriedades da madeira de eucalipto provenientes de diferentes SAF's para fins energéticos. Foram avaliados três SAF's, com espaçamentos entre árvores de $9 \times 1 \mathrm{~m}$ (Sistema 1), de $8 \times 3 \mathrm{~m}$ (Sistema 2) e de $12 \times 3 \mathrm{~m}$ (Sistema 3). Em cada Sistema, foram selecionadas três árvores-amostra por classe de DAP para a determinação do volume individual, biomassa seca, densidade básica, teor de carbono e Poder Calorífico Superior (PCS) da madeira. O Sistema 1 apresentou árvores com maior altura, enquanto no Sistema 3 foram observadas árvores com maior diâmetro. Não foi verificada diferença significativa no teor de carbono entre os Sistemas estudados, com valor médio igual a $52,2 \%$. A densidade básica variou de 0,40 a $0,43 \mathrm{~g} \mathrm{~cm}^{-3}$ sendo verificado os maiores valores para os Sistemas 2 e 3 . O PCS variou de 4576 a $4631 \mathrm{kcal} \mathrm{kg}^{-1}$, com maior valor para o Sistema 1. As maiores estimativas de Incremento Médio Anual $\left(43,01 \mathrm{~m}^{3} \mathrm{ha}^{-1} \mathrm{ano}^{-1}\right)$, biomassa seca da madeira $\left(17,53 \mathrm{t} \mathrm{ha}^{-1} \mathrm{ano}^{-1}\right)$, carbono $\left(9,16 \mathrm{t} \mathrm{ha}^{-1} \mathrm{ano}^{-1}\right)$ e energia disponível $\left(69,82 \mathrm{GWh} \mathrm{ha}^{-1}\right.$ ano $\left.^{-1}\right)$ foram obtidas para o Sistema 1, em decorrência do maior número de árvores por hectare, em comparação aos demais Sistemas. Conclui-se que os Sistemas Agroflorestais avaliados apresentam potencial para a produção de biomassa seca de madeira para fins energéticos, com melhores valores para o Sistema 1 que apresenta maior número de árvores por hectare.
\end{abstract}

Palavras-chave: Integração lavoura-pecuária-floresta, Energia, Espaçamento.

\begin{abstract}
Eucalyptus wood produced in Agroforestry Systems (AFS) can be used for various purposes such as firewood, fence posts, lumber and charcoal. When it is used for energy purposes, mainly in the form of firewood, it is obtained by thinning and presents smaller diameters. The aim of this work was to estimate the volumetric production and determine the properties of eucalyptus wood from different AFSs for energy purposes. Three AFSs with tree spacing of $9 \times 1 \mathrm{~m}$ (System 1), of $8 \times 3 \mathrm{~m}$ (System 2), and of $12 \times 3 \mathrm{~m}$ (System 3 ) were evaluated. In each System, we selected three sample trees per DBH class for individual volume determination, basic density, dry wood biomass, carbon content, and higher heating value (HHV) of wood. System 1 presented trees with larger height and the System 3 with larger diameter. There was no significant difference in carbon content among the Systems studied, with an average value of $52.2 \%$. The basic density ranged from 0.40 to $0.43 \mathrm{~g} \mathrm{~cm}^{-3}$, with the highest values found in Systems 2 and 3. The PCS ranged from 4576 to $4631 \mathrm{kcal} \mathrm{kg}^{-1}$, with the highest value for the System 1.The first System yielded higher HHV and lower density compared to other Systems. The highest estimates of Mean Annual Increment $\left(43.01 \mathrm{~m}^{3} \mathrm{ha}^{-1} \mathrm{yr}^{-1}\right)$, dry wood biomass (17.53 tha- $\left.\mathrm{yr}^{-1}\right)$, carbon $\left(9.16 \mathrm{t} \mathrm{ha}^{-1} \mathrm{yr}^{-1}\right)$ and available energy (69.82 $\left.\mathrm{GWh} \mathrm{ha}^{-1} \mathrm{yr}^{-1}\right)$ were

1Professor Adjunto do Departamento de Engenharia Florestal. UFV - Universidade Federal de Viçosa. Av. PH Rolfs s/n 36571-000 - Viçosa, MG, Brasil. E-mail: carlos.eleto@yahoo.com.br

${ }^{2}$ Professor(a) Assistente. Universidade Federal de Mato Grosso / Faculdade de Engenharia Florestal. Caixa-postal: 78060900 - 78060900 - Cuiabá, MT - Brasil. E-mail: aylsoncosta@gmail.com; babicorradi@gmail.com

${ }^{3}$ Professor Associado do Departamento de Engenharia Florestal. UFV - Universidade Federal de Viçosa. Campus Universitario s/n - 36571-000 - Vicosa, MG, Brasil. E-mail: jacovine@ufv.br

${ }^{4}$ Professor(a) Associado(a) do Departamento de Engenharia Florestal. UFV - Universidade Federal de Viçosa. Av. PH Rolfs s/n - 36571-000 - Viçosa, MG, Brasil. E-mail: snolasco@ufv.br; cassiacarneiro1@gmail.com

${ }^{5}$ Mestrando em Ciência Florestal. UFV - Universidade Federal de Viçosa. Av. PH Rolfs s/n - 36571-000 - Viçosa, MG, Brasil. E-mail: caiomiquelino@yahoo.com.br
\end{abstract}


obtained in System 1, due to greater number of trees per hectare when compared to the other Systems. We conclude that the Agroforestry Systems evaluated show potential production of dry wood biomass for energy purposes, with better values for the System 1 that has a higher number of trees per hectare.

Keywords: Crop-livestock-forest integration, Energy, Tree Spacing

\section{INTRODUÇÃO}

Nos últimos anos, o Governo Federal tem implementado programas de incentivo à adoção de Sistemas Agrissilvipastoril e Silvipastoril, por meio do Programa Nacional de Fortalecimento da Agricultura Familiar - PRONAF, modalidades Floresta e Eco, e do Programa Agricultura de Baixo Carbono (ABC) (BRASIL, 2012). Nestes modelos de Sistemas Agroflorestais, culturas agrícolas, árvores, pastagens e animais são integrados visando potencializar interações ecológicas e econômicas resultantes, consistindo em uma alternativa para agricultores interessados na produção de madeira.

No Brasil, espécies do gênero Eucalyptus têm sido as mais utilizadas na composição desses Sistemas, diante do potencial de crescimento, adaptação a diferentes condições ecológicas e usos de sua madeira, aliados ao conhecimento silvicultural e genético adquirido nas últimas décadas (OLIVEIRA NETO et al., 2007).

O volume de madeira produzido e sua qualidade podem ser influenciados pelos arranjos espaciais adotados nesses Sistemas, uma vez que são mais amplos, visando otimizar a disponibilidade de recursos de crescimento para outras espécies que os compõem (FONTAN et al., 2011; GONÇALVES et al., 2004).

Os espaçamentos amplos do componente arbóreo em Sistemas Agrissilvipastoris e Silvipastoris favorecem o crescimento em diâmetro e volume individual das árvores (LEITE et al. 2006) e são fundamentais para a satisfatória produção das espécies forrageiras (OLIVEIRA NETO; PAIVA, 2010; PRASAD et al., 2010). Quando espaçamentos menores, entre plantas, nas linhas de plantios, ou fileiras múltiplas adensadas, são adotados visando elevar o número de árvores por área, desbastes tornam-se necessários quando se planeja a produção de madeira de qualidade para serraria (FRANKE; FURTADO 2001; OLIVEIRA NETO; PAIVA, 2010). A madeira obtida pelo desbaste normalmente apresenta dimensões reduzidas, indicando a utilização para fins energéticos, sobretudo na forma de lenha.

Estudos sobre a viabilidade econômica destes Sistemas normalmente consideram apenas o crescimento das árvores e a produção volumétrica de madeira (CORDEIRO; SILVA, 2010; PAULA et al., 2013), desconsiderando suas características tecnológicas, que podem variar de acordo com o espaçamento de plantio e idade de corte das árvores. Sendo assim, estimativas da densidade básica, do teor de carbono e de energia, são importantes para uma análise mais consistente sobre a viabilidade da produção de madeira nestes Sistemas, conforme destacado por Santos et al. (2012).

Diante disto, este estudo teve como objetivo avaliar a produção de biomassa seca e as propriedades da madeira de eucalipto para fins energéticos produzida em Sistemas Agrissilvipastoril e Silvipastoril.

\section{MATERIAL E MÉTODOS}

\section{Localização e descrição do experimento em campo}

Foram avaliados três Sistemas, localizados na zona rural do município de Viçosa $\left(20^{\circ} 45^{\prime} \mathrm{S}\right.$ e 4251' W), MG, que apresenta altitude média de 650m e clima, segundo a classificação de Köppen, do tipo Cwa, mesotérmico, com inverno seco e verão chuvoso (ALVARES et al., 2013). A precipitação média anual é de aproximadamente $1.200 \mathrm{~mm}$ e a temperatura média anual de $19,4^{\circ} \mathrm{C}$, com mínima de $14,8^{\circ} \mathrm{C}$ e máxima de $26,4^{\circ} \mathrm{C}$ (RAMOS et al., 2009).

As informações sobre o histórico dos Sistemas e adubações realizadas foram coletadas por meio de entrevistas aos produtores rurais. A composição dos Sistemas avaliados são descritos a seguir e na Tabela 1:

- Sistema 1: Pasto (Urochloa decumbens) + eucalipto (Eucalyptus urophylla X Eucalyptus grandis)

Para o plantio dos clones de eucalipto aplicou-se 0,2 kg NPK (06-30-06) cova $^{-1}$ e, após tês me- 
ses, foi realizada uma adubação de cobertura de 0,16 kg NPK (20-05-20) cova $^{-1}$. Para a pastagem aplicou-se $100 \mathrm{~kg}$ ureia ha ${ }^{-1}$ ano $^{-1}$.

- Sistema 2: Feijão (Phaseolus vulgaris) + pasto (Urochloa decumbens) + eucalipto (E. urophylla X E. grandis)

No Sistema 2, foi realizada dessecação em área total com herbicida glyphosate na dose de $1 \mathrm{~L}$ ha${ }^{1}$, antes da implantação do Sistema. Os plantios do capim-braquiária e do feijão foram realizados simultaneamente, com auxílio de plantadeira com tração animal específica para o plantio direto. Para a cultura do feijão, aplicou-se $300 \mathrm{~kg}$ NPK (08-28-16) ha-1 e $200 \mathrm{~kg}^{-1}$ ureia ha $\mathrm{ha}^{-1} \mathrm{em}$ cobertura. Após a colheita do feijão foi mantido somente o pasto com eucalipto, aplicando-se $100 \mathrm{~kg} \mathrm{ureia} \mathrm{ha-1}^{-1}$ ano $^{-1}$. O plantio e a manutenção do eucalipto seguiram os mesmos procedimentos do Sistema 1.

- Sistema 3: Pasto (Urochloa decumbens) + eucalipto (E. urophylla X E. grandis)

No plantio dos clones de eucalipto foi aplicado $0,2 \mathrm{~kg} \mathrm{NPK} \mathrm{(06-30-06)}$ cova $^{-1}$ e realizadas quatro adubações de cobertura com 0,05 kg NPK (20-05-20) planta ${ }^{-1}, 60$ dias após o plantio, 0,1 kg NPK (20-05-20) planta $^{-1} 120$ dias após, 0,15 kg NPK (20-05-20) planta ${ }^{-1}$, aos 10 meses e 0,1 kg KCl plan$\mathrm{ta}^{-1}$, aos 18 meses. Para o capim-braquiária, que já estava estabelecido, aplicou-se, no ano de 2011, $100 \mathrm{~kg}$ NPK (20-05-20) ha-1.

Tabela 1. Resumo dos Sistemas Silvipastoris (1 e 3) e Agrissilvipastoril (2).

Table 1. Summary of Silvipastoral (1 and 3) and Agrosilvopastoral(2) Systems.

\begin{tabular}{lcccccc}
\hline Sistema & $\begin{array}{c}\text { Cultura } \\
\text { agrícola }\end{array}$ & Pastagem & $\begin{array}{c}\text { Idade } \\
\text { (meses) }\end{array}$ & $\begin{array}{c}\text { Área } \\
\text { (ha) }\end{array}$ & $\begin{array}{c}\text { Espaçamento do } \\
\text { eucalipto (m) }\end{array}$ & $\begin{array}{c}\text { Densidade } \\
\text { (árvores ha-1) }^{-1}\end{array}$ \\
\hline 1 & - & Braquiária & 32 & 0,55 & $9 \times 1$ & 1.111 \\
2 & Feijão & Braquiária & 32 & 0,72 & $8 \times 3$ & 416 \\
3 & - & Braquiária & 32 & 3,48 & $12 \times 3$ & 277 \\
\hline
\end{tabular}

\section{Amostragem e coleta de dados}

Após 32 meses do plantio, foi realizado o censo florestal nos Sistemas avaliados, mensurando o CAP (circunferência com casca a 1,30m de altura) e as alturas total e comercial de todas as árvores. Foi realizada a caracterização da distribuição diamétrica do povoamento florestal, sendo selecionadas três árvores-amostra por classe de DAP, em cada Sistema.

Para a determinação do volume do fuste com e sem casca, de cada árvore amostra, realizou-se o corte e foram mensurados os diâmetros com casca e as espessuras da casca nas alturas de 0,3m, $0,7 \mathrm{~m}, 1,3 \mathrm{~m}$ e, a partir desta altura, a cada 2 metros, até um diâmetro mínimo com casca de $3 \mathrm{~cm}$ (altura comercial).

Além disso, para cada árvore amostrada, foram retirados discos de 2,5 $\mathrm{cm}$ de espessura a partir de 0 (base), 25\%, 50\%, 75\% e 100\% da altura comercial do tronco e a 1,3 m. Cada disco foi subdividido em quatro partes, em forma de cunha, passando pela medula. Duas cunhas opostas foram utilizadas para determinação da densidade básica da madeira, e usou-se as outras duas cunhas restantes para as análises da composição elementar e poder calorífico superior.

\section{Propriedades da Madeira e Estimativas}

Para a determinação da densidade básica da madeira foi utilizado o método de imersão em água, descrito por Vital (1984) e pela NBR 11941 (ABNT, 2003), utilizando-se uma balança hidrostática. Os valores médios foram calculados a partir da média aritmética das densidades das respectivas cunhas.

O poder calorífico superior da madeira foi determinado segundo a norma da NBR 8633 (ABNT, 1984), utilizando-se uma bomba calorimétrica adiabática.

O teor de $\mathrm{C}$ na madeira foi determinado pelo método da combustão seca, utilizando-se um determinador elementar de C, H e N (TruSpec Micro CHN LECO Corp., St. Joseph, MI).

A biomassa seca de madeira sem casca foi obtida multiplicando-se o Incremento Médio Anual Volumétrico (IMA) da madeira sem casca pela densidade básica da madeira, conforme a equação (1):

$$
M S M=I M A \times D b m(1)
$$

em que:

$\mathrm{MSM}=$ biomassa seca de madeira $\left(\mathrm{t} \mathrm{ha}^{-1} \mathrm{ano}^{-1}\right)$; 
$\mathrm{IMA}=$ volume da madeira sem casca $\left(\mathrm{m}^{3} \mathrm{ha}^{-1} \mathrm{ano}^{-1}\right) ; \mathrm{e}$

Dbm = densidade básica da madeira $\left(\mathrm{kg} \mathrm{m}^{-3}\right)$.

O estoque de carbono foi obtido multiplicando-se a biomassa seca de madeira pelo teor de carbono na madeira, conforme a equação (2).

$$
M C=\operatorname{MSM} x(2)
$$

em que:

$\mathrm{MC}=$ Carbono $\left(\mathrm{t} \mathrm{ha}^{-1} \mathrm{ano}^{-1}\right) ;$

$\mathrm{MSM}=$ biomassa seca da madeira $\left(\mathrm{t} \mathrm{ha}^{-1} \mathrm{ano}^{-1}\right) ; \mathrm{e}$

$\mathrm{C}(\%)=$ teor de carbono.

Para o cálculo da quantidade de energia, expressa em kWh, multiplicou-se a biomassa de matéria seca da madeira pelo poder calorífico superior, fazendo-se a equivalência de $10^{-6} \mathrm{GWh}$ a $859,85 \mathrm{kcal}$, calculado pela equação (3).

\section{Energia Disponível $=$ MSMx PCS mad (3)}

em que:

Energia Disponível $=$ energia por hectare $\mathrm{ano}^{-1}\left(\mathrm{GWh} \mathrm{ha}^{-1} \mathrm{ano}^{-1}\right)$;

$\mathrm{MSM}=$ biomassa seca da madeira $\left(\mathrm{t} \mathrm{ha}^{-1} \mathrm{ano}^{-1}\right) ; \mathrm{e}$

PCS mad = poder calorífico superior da madeira $\left(\mathrm{kcal} \mathrm{kg}^{-1}\right)$.

\section{Análise Estatística dos Dados}

Os resultados foram interpretados com o auxílio da análise de variância (ANOVA), aplicando-se o teste $\mathrm{F}$, e quando estabelecidas diferenças significativas, as médias foram comparadas pelo teste de Tukey a $95 \%$ de probabilidade.

As análises estatísticas foram realizadas com o auxílio do software R.

\section{RESULTADOS E DISCUSSÃO}

\section{Características de crescimento}

As características das árvores-amostras dos três Sistemas, por classe diamétrica, estão apresentadas na Tabela 2.

As árvores no Sistema 1, cujo espaçamento de plantio foi de $9 \times 1 \mathrm{~m}$, apresentaram maiores alturas, se comparadas com aquelas dos Sistemas 2 e 3, com espaçamentos de $8 \times 3 \mathrm{~m}$ e 12 x 3m, respectivamente. Esse maior crescimento em altura das árvores do Sistema 1 pode ser explicado pela competição entre as plantas, pois este Sistema tem uma menor área útil por planta $\left(9 \mathrm{~m}^{2}\right)$, se comparado com o Sistema $2\left(24 \mathrm{~m}^{2}\right)$ e com o $3\left(36 \mathrm{~m}^{2}\right)$. Além disso, nos Sistemas 2 e 3, a distância entre plantas na linha é de três metros, enquanto no Sistema 1 é um metro.

Essa competição fez com que as árvores tivessem um menor crescimento em diâmetro e um aumento na altura total. Nesse Sistema há também um maior número de árvores dominadas, ou seja, classes diamétricas menores (Tabela 2), demostrando que o processo de competição promoveu crescimento mais desuniforme das árvores.

Em nível de povoamento, devido a densidade populacional, o Sistema 1 apresentou um volume de madeira com casca $61 \%$ maior que o Sistema 2 e 79\% maior que o Sistema 3. Já para o volume sem casca o Sistema 1 foi $57 \%$ e 78\% maior que os Sistemas 2 e 3, respectivamente (Tabela 3). Porém, acredita-se que com o aumento da idade dos plantios, os Sistemas com espaçamentos mais amplos terão um maior potencial de crescimento das árvores e, como consequência, um volume mais próximo daqueles Sistemas com espaçamentos menores, que terão maior competição por recursos de crescimento, podendo ocorrer inclusive a morte de árvores dominadas (BERGER, 2000; SANTOS, 2011). 
Tabela 2. Características de crescimento das árvores-amostras por classe diamétrica, em cada SistemaAgroflorestal.

Table 2. Growth characteristics of sample trees by diameter class in each Agroforestry Systems.

\begin{tabular}{|c|c|c|c|c|c|c|c|c|}
\hline \multicolumn{9}{|c|}{ Sistema 1 (9 x 1m) } \\
\hline Centro de Classe & $\mathrm{Ht}$ & Hc & DAPcc & DAPsc & Vcc & Vsc & Vcasca & $\%$ Casca \\
\hline 6,25 & 10,09 & 7,83 & 6,79 & 6,35 & 0,03 & 0,02 & 0,003 & 13,53 \\
\hline 8,75 & 14,29 & 11,63 & 9,18 & 8,48 & 0,08 & 0,06 & 0,01 & 13,88 \\
\hline 11,25 & 16,05 & 13,35 & 11,6 & 10,7 & 0,12 & 0,10 & 0,02 & 15,66 \\
\hline 13,75 & 18,47 & 15,92 & 14,5 & 13,51 & 0,21 & 0,18 & 0,03 & 13,46 \\
\hline 16,25 & 19,2 & 16,81 & 15,68 & 14,61 & 0,26 & 0,22 & 0,03 & 13,36 \\
\hline 18,75 & - & - & - & - & - & - & - & - \\
\hline Média Ponderada & 16,59 & 14,04 & 12,53 & 11,65 & 0,16 & 0,14 & 0,02 & 14,06 \\
\hline \multicolumn{9}{|c|}{ Sistema $2(8 \times 3 \mathrm{~m})$} \\
\hline Centro de Classe & $\mathrm{Ht}$ & $\mathrm{Hc}$ & DAPcc & DAPsc & Vcc & Vsc & Vcasca & $\%$ Casca \\
\hline 6,25 & - & - & - & - & - & - & - & - \\
\hline 8,75 & 10,28 & 7,70 & 9,00 & 7,98 & 0,05 & 0,04 & 0,01 & 20,76 \\
\hline 11,25 & 13,55 & 11,04 & 12,07 & 10,83 & 0,11 & 0,09 & 0,02 & 17,82 \\
\hline 13,75 & 15,84 & 13,44 & 14,63 & 13,38 & 0,19 & 0,15 & 0,03 & 16,61 \\
\hline 16,25 & 18,15 & 15,93 & 17,52 & 16,32 & 0,29 & 0,24 & 0,04 & 14,47 \\
\hline 18,75 & - & - & - & - & - & - & - & - \\
\hline Média Ponderada & 15,03 & 12,61 & 13,84 & 12,62 & 0,17 & 0,14 & 0,03 & 16,90 \\
\hline \multicolumn{9}{|c|}{ Sistema 3 (12 x 3m) } \\
\hline Centro de Classe & $\mathrm{Ht}$ & Hc & DAPcc & DAPsc & Vcc & Vsc & Vcasca & $\%$ Casca \\
\hline 6,25 & 6,18 & 4,07 & 6,92 & 6,34 & 0,02 & 0,01 & 0,003 & 17,36 \\
\hline 8,75 & 9,97 & 7,44 & 9,68 & 8,67 & 0,06 & 0,05 & 0,01 & 19,91 \\
\hline 11,25 & 12,0 & 9,92 & 11,72 & 10,66 & 0,10 & 0,08 & 0,02 & 18,01 \\
\hline 13,75 & 13,5 & 11,21 & 13,97 & 12,52 & 0,15 & 0,12 & 0,03 & 19,98 \\
\hline 16,25 & 15,25 & 13,21 & 16,89 & 15,3 & 0,24 & 0,2 & 0,04 & 16,50 \\
\hline 18,75 & 17,89 & 15,91 & 20,8 & 19,59 & 0,24 & 0,21 & 0,03 & 13,40 \\
\hline Média Ponderada & 12,49 & 10,28 & 12,84 & 11,61 & 0,13 & 0,11 & 0,02 & 18,55 \\
\hline
\end{tabular}

Centro de Classe $=$ centro da classe diamétrica $(\mathrm{cm}), \mathrm{Ht}$ e $\mathrm{Hc}=$ altura total e comercial $(\mathrm{m})$, DAPcc e sc = diâmetro a $\mathrm{I}, 3 \mathrm{~m}$ de altura do solo come sem casca $(\mathrm{cm})$, Vcc e sc $=$ volume com e sem casca por árvore-amostra $\left(\mathrm{m}^{3}\right)$, Vcasca $=$ volume de casca $\left(\mathrm{m}^{3}\right), \%$ Casca $=$ percentagem de casca

A porcentagem de casca nos troncos das árvores nos Sistemas variou entre 16 e 22\%, com maior tendência no Sistema 1 (Tabela 3). Oliveira et al. (1999) avaliando a porcentagem de casca para sete diferentes espécies de eucalipto, com espaçamento convencional (2x3 m e 3x3 m), em Anhembi SP, obtiveram uma porcentagem de 10,82\% e 12,75\% para E. grandis e E. urophylla, respectivamente, valores inferiores ao encontrado na pesquisa.

Tabela 3. Volume com casca e sem casca, e porcentagem de casca, nos Sistemas Agroflorestais.

Table 3. Volume inside and outside bark, and bark percentage, in the Agroforestry Systems.

\begin{tabular}{lccc}
\hline Sistema & Volume com Casca $\left(\mathbf{m}^{\mathbf{3}} \mathbf{h a}^{-1}\right)$ & Volume sem casca $\left(\mathbf{m}^{\mathbf{3}} \mathbf{h a}^{-1}\right)$ & \% Casca \\
\hline 1 & 148,23 & 114,66 & 22,65 \\
2 & 58,27 & 48,82 & 16,22 \\
3 & 31,27 & 25,80 & 17,49 \\
\hline
\end{tabular}

\section{Propriedades da Madeira}

a) Densidade da madeira

A densidade básica média da madeira de eucalipto (Eucalyptus urophylla X Eucalyptus grandis) proveniente dos Sistemas 2 e 3 foi aproximadamente 7\% superior à densidade da madeira do Sistema 1 (Tabela 4).

Tabela 4. Densidade básica média da madeira de eucalipto, em g. $\mathrm{cm}^{-3}$, por classe de diâmetro, nos Sistemas Agroflorestais.

Table 4. Mean basic density of wood from Eucalyptus, in ${\mathrm{g} . \mathrm{cm}^{-3}}^{3}$, by diameter class in the Agroforestry Systems.

\begin{tabular}{lccccccc}
\hline \multirow{2}{*}{ Sistema } & \multicolumn{7}{c}{ Centro de Classe $(\mathbf{c m})$} \\
\cline { 2 - 8 } & $\mathbf{6 , 2 5}$ & $\mathbf{8 , 7 5}$ & $\mathbf{1 1 , 2 5}$ & $\mathbf{1 3 , 7 5}$ & $\mathbf{1 6 , 2 5}$ & $\mathbf{1 8 , 7 5}$ & Média \\
\hline $1(9 \times 1 \mathrm{~m})$ & 0,34 & 0,40 & 0,37 & 0,43 & 0,40 & - & $0,40 \mathrm{~b}$ \\
$2(8 \times 3 \mathrm{~m})$ & - & 0,41 & 0,44 & 0,40 & 0,43 & - & $0,43 \mathrm{a}$ \\
$3(12 \times 3 \mathrm{~m})$ & 0,40 & 0,41 & 0,42 & 0,45 & 0,45 & 0,44 & $0,43 \mathrm{a}$ \\
\hline
\end{tabular}

Médias seguidas de mesma letra não diferem estatisticamente, pelo teste de Tukey, a 5\% de significância. 
Os maiores valores médios da densidade foram encontrados nos sistemas cujo eucalipto foi plantado em maiores espaçamentos. Esta variação na densidade básica da madeira pode estar relacionada à disponibilidade de fotoassimilados para o crescimento secundário das árvores. Isso porque plantios com menores espaçamentos têm uma densidade populacional maior, o que irá provocar maior competição entre plantas por água, luz e nutrientes. Os resultados encontrados estão de acordo com DeBell et al. (2001), Berger et al. (2002), Haselein et al. (2002) e Rocha (2011), os quais verificaram que o aumento do espaçamento resulta em uma maior densidade básica da madeira de eucalipto.

De acordo com Souza et al. (2008), a competição por nutrientes ocasiona redução do incremento de biomassa das plantas. Desta forma, árvores plantadas em maiores espaçamentos terão maior disponibilidade de recursos de crescimento e aumento da produção de biomassa (OLIVEIRA NETO et al., 2003). Tal fato irá contribuir para o aumento da capacidade de armazenar carbono, retirando-o da atmosfera e sintetizando celulose, hemiceluloses e lignina, componentes da parede celular.

Outros autores, porém, citam que existe uma diminuição da densidade básica da madeira com o aumento do espaçamento, ou ainda, que a densidade não é afetada pelo espaçamento (WASHUSEN et al., 2005; TREVISAN et al., 2012a,b). Para Elliott (1970), as divergências sobre a influência do ritmo de crescimento sobre a densidade da madeira têm sido atribuídas às seguintes causas: padrões de amostragem; idade das árvores amostradas em associação com o grau de competição entre elas; disponibilidade de água, também associada com o grau de competição; influência das características da copa na época da colheita.

Outro fator que pode ter contribuído para as maiores densidades da madeira dos Sistemas 2 e 3 foram os maiores níveis de adubação nesses sistemas. De acordo com Berger et al. (2002), há um acréscimo gradual na densidade da madeira de eucalipto em função do aumento da área útil e das doses de adubo aplicadas. Embora tenha-se verificado essas diferenças significativas da densidade básica, Zobel (1984) relata que o efeito da adubação nas angiospermas dicotiledôneas com poros difusos, como as espécies do gênero Eucalyptus, provoca resultados conflitantes sobre essa característica da madeira.

Em relação aos centros de classe, observou-se uma tendência de aumento de densidade da madeira com o aumento do DAP das árvores. Tal fato também foi observado por Manfredi e Barrichelo (1985); Trugilho et al. (2010). Manfredi e Barichelo (1985), estudando as propriedades da madeira de povoamentos comerciais de E. grandis e E. saligna, verificaram que as árvores dominadas apresentam taxas inferiores de fotossíntese e menor disponibilidade de fotoassimilados, e isso pode ter sido a causa determinante de uma menor densidade básica dessas árvores, nos sistemas 1, 2 e 3 . Ferreira e Kageyama (1978), ao avaliarem a madeira de E. saligna, também verificaram que árvores mais vigorosas apresentaram valores de densidade básica maiores quando comparadas com árvores menos vigorosas.

\section{b) Teor de carbono}

Em relação aos valores médios de carbono (Figura 1), não foram observadas diferenças significativas entre os Sistemas, a 95\% de probabilidade. Tal fato demonstra que o espaçamento e a associação de culturas agrícolas com o eucalipto não influenciaram no teor de carbono da madeira.

De modo geral, constatou-se, em diversos trabalhos encontrados na literatura, como os de Pereira et al. (2013), Protásio et al. (2014) e Santos et al. (2011), que não há diferenças em relação à percentagem de carbono na madeira de diferentes espécies do gênero Eucalyptus. Além disso, esses mesmos autores observaram que a madeira é constituída de, aproximadamente, 50\% de carbono, valor que está em conformidades com o verificado no presente trabalho.

\section{c) Poder calorífico}

O poder calorífico superior (PCS) da madeira do Sistema 1 ( $9 \times 1 \mathrm{~m})$ foi estatisticamente superior aos demais (Figura 2). Essas diferenças podem ser causadas por variações nos teores de extrativos e lignina que afetam diretamente o poder calorífico da madeira (PEREIRA et al., 2013; SANTOS et al., 2011).

O PCS da madeira apresenta variação dentro do gênero Eucalyptus, o que pôde ser observado no presente trabalho e nos trabalhos de Oliveira et al. (2010), Santos et al. (2011), Protásio et al. (2014). 


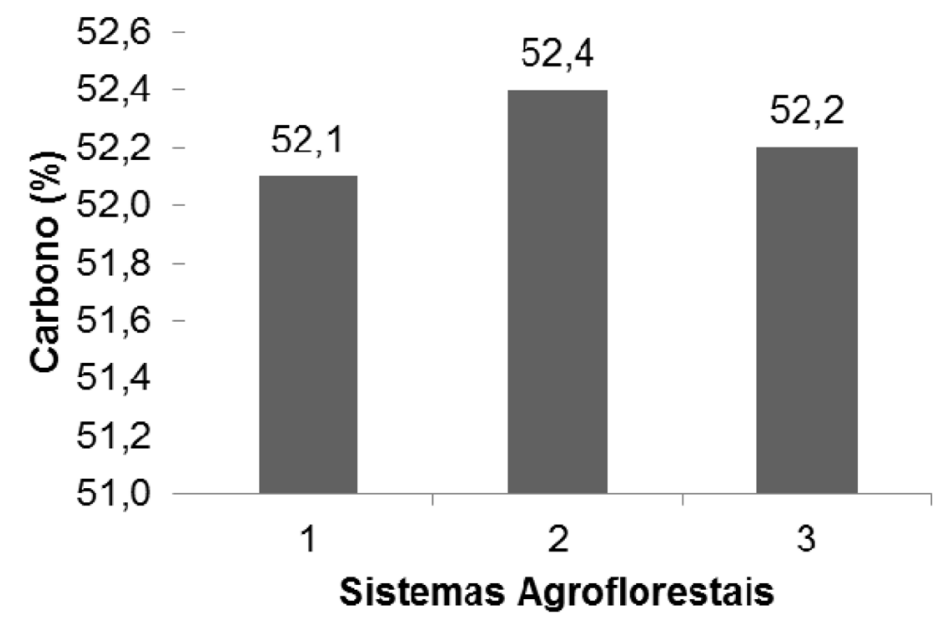

Figura 1. Teor de carbono na madeira nos Sistemas Agroflorestais.

Figure 1. Wood carbon content in the Agroforestry Systems.

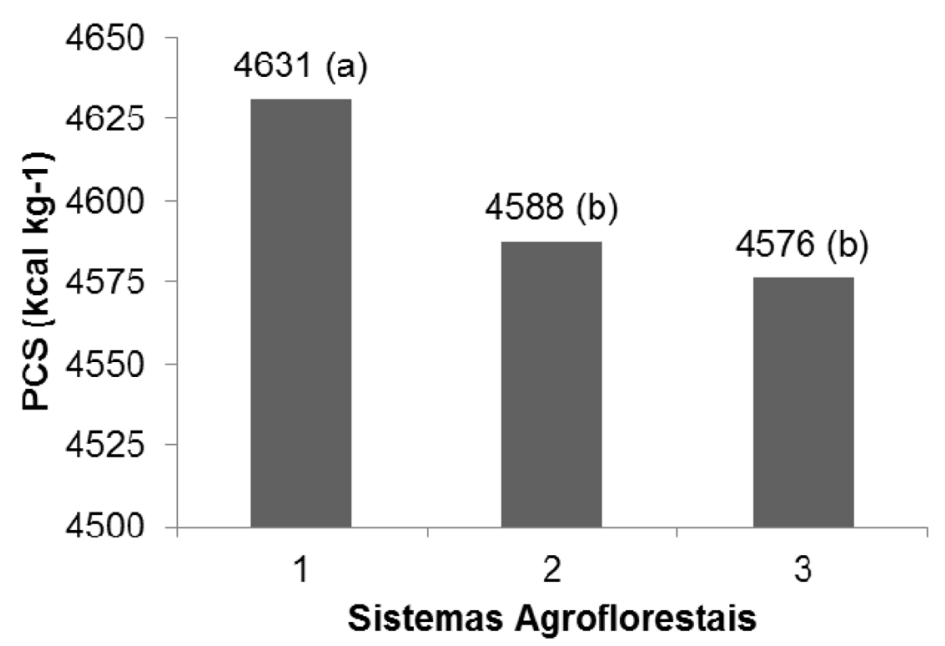

Médias seguidas de mesma letra não diferem estatisticamente, pelo teste de Tukey, a $5 \%$ de significância.

Figura 2. Poder calorífico superior da madeira sem casca nos Sistemas Agroflorestais.

Figure 2. Higher heating value of wood inside bark in the Agroforestry Systems.

\section{Incremento médio anual, Biomassa seca de madeira, Carbono e Energia disponível}

Em relação ao incremento médio anual (Tabela 5), observa-se que o Sistema 1 foi superior aos Sistemas 2 e 3 . A diferença encontrada nos valores de IMA está relacionada ao número de árvores por hectare, considerando os espaçamentos adotados em cada Sistema. Os IMAs dos Sistemas 2 e 3 são, respectivamente, $57,5 \%$ e $77,5 \%$ menores que o IMA do Sistema 1 . Isto porque o número de árvores por hectare dos Sistemas 2 e 3 é 62,5\% e 75\% menores que no Sistema 1, respectivamente, e a variação do volume médio de madeira por árvore é similar para os Sistemas 1 e 2. Já o Sistema 3, obteve o menor IMA por apresentar o menor número de árvores por hectare, além do menor volume médio de madeira por árvore. Vale salientar que se não houver uma intervenção, a partir de desbaste, poderá ocorrer uma competição entre os indivíduos dos Sistemas mais adensados, e como consequência a diminuição do IMA.

Santana (2009) verificou IMA igual a $37,36 \mathrm{~m}^{3} \mathrm{ha}^{-1} \mathrm{ano}^{-1}$ para um clone de E. grandis x E. urophylla, aos 34 meses, em espaçamento 3,0 x 2,8 m, no município de Bom Sucesso, MG. Este IMA aproxima-se daquele encontrado para o Sistema 1, cujas áreas úteis por planta são similares, porém com arranjos espaciais distintos. 
Tabela 5. Valores médios de incremento médio anual (IMA) e das estimativas de biomassa de matéria seca de madeira, carbono e energia nos Sistemas Agroflorestais.

Table 5. Mean values of mean annual increment (MAI) and of biomass estimates of dry wood, carbon and energy in the Agroforestry Systems.

\begin{tabular}{|c|c|c|c|c|c|c|c|c|}
\hline \multirow{2}{*}{ Estimativa } & \multirow{2}{*}{ Sistema } & \multicolumn{6}{|c|}{ Centro de classe $(\mathrm{cm})$} & \multirow{2}{*}{ Total } \\
\hline & & 6,25 & 8,75 & 11,25 & 13,75 & 16,25 & 18,75 & \\
\hline & 1 & 1,00 & 1,45 & 9,19 & 25,33 & 6,05 & - & 43,01 \\
\hline \multirow[t]{3}{*}{ IMA $\left(\mathrm{m}^{3} \mathrm{ha}^{-1} \mathrm{ano}^{-1}\right)$} & 2 & - & 0,38 & 4,49 & 6,70 & 6,74 & - & 18,31 \\
\hline & 3 & 0,08 & 0,56 & 2,35 & 3,60 & 2,68 & 0,40 & 9,68 \\
\hline & 1 & 0,30 & 0,58 & 3,42 & 10,79 & 2,41 & - & 17,53 \\
\hline \multirow[t]{3}{*}{ Biomassa seca da madeira ( $\left.\mathrm{t} \mathrm{ha}^{-1} \mathrm{ano}^{-1}\right)$} & 2 & - & 0,16 & 1,97 & 2,71 & 2,92 & - & 7,75 \\
\hline & 3 & 0,03 & 0,23 & 1,00 & 1,62 & 1,20 & 0,18 & 4,26 \\
\hline & 1 & 0,20 & 0,30 & 1,79 & 5,63 & 1,26 & - & 9,16 \\
\hline \multirow[t]{3}{*}{ Carbono (t ha ${ }^{-1} \mathrm{ano}^{-1}$ ) } & 2 & - & 0,08 & 1,03 & 1,41 & 1,52 & - & 4,04 \\
\hline & 3 & 0,02 & 0,12 & 0,52 & 0,85 & 0,63 & 0,09 & 2,23 \\
\hline & 1 & 1,33 & 2,30 & 13,62 & 42,97 & 9,61 & - & 69,82 \\
\hline \multirow[t]{2}{*}{ Energia disponível (GWh ha-1ano-1) } & 2 & - & 0,63 & 7,77 & 10,67 & 11,51 & - & 30,58 \\
\hline & 3 & 0,13 & 0,92 & 3,93 & 6,39 & 4,71 & 0,69 & 16,77 \\
\hline
\end{tabular}

Observa-se que a produção de biomassa seca, carbono e energia no Sistema 1 foram maiores que nos Sistemas 2 e 3 (Tabela 6). Isso se deve aos menores valores de IMA obtidos à medida que se aumentou o espaçamento entre árvores nos Sistemas. Em relação às classes diamétricas de um mesmo Sistema, as diferenças nos IMAs resultaram em variações expressivas nas estimativas de biomassa seca, carbono e energia por centro de classe (Tabela 6).

Quanto à biomassa seca por hectare ano $^{-1}$ (Tabela 5), pode-se observar que o maior valor foi observado no Sistema 1, o que está relacionado ao maior número de árvores e portanto, maior volume de madeira por hectare. A maior densidade básica da madeira de eucalipto nos Sistemas 2 e 3 não foi suficiente para compensar o maior IMA do Sistema 1. As biomassas secas dos Sistemas $2 \mathrm{e}$ 3 foram $56 \%$ e $76 \%$, respectivamente, menores que no Sistema 1.

O valor médio de biomassa seca no Sistema 1, cuja área útil por árvore se assemelha a plantios florestais em monocultura que visam a produção de energia, foi de 17,53 $\mathrm{t} \mathrm{ha}^{-1} \mathrm{ano}^{-1}$. Esse valor se assemelha ao encontrado por Rocha (2011), para clone de E. grandis $\mathrm{x}$ E.camaldulensis, aos 48 meses de idade, em espaçamento 3 x 3 m, no município de Itamarandiba, MG, que verificou valor de biomassa seca de madeira igual a 17,66 t ha-1 $\mathrm{ano}^{-1}$. Santana (2009) encontrou valor médio de 14,33 t ha $^{-1}$ ano $^{-1}$ para um clone de eucalipto aos 34 meses, em espaçamento 3,0 x 2,8 m, no município de Bom Sucesso, MG.

Como não houve diferenças significativas do teor de carbono na madeira proveniente dos Sistemas avaliados, o que mais influenciou a massa de carbono foi a biomassa seca da madeira (Tabela 5). Portanto, como a biomassa seca de madeira do Sistema 1 foi maior, a massa de carbono estocada na madeira deste Sistema também foi superior aos demais. Esse comportamento evidencia que maiores IMAs aumentam o estoque de carbono no Sistema Agroflorestal.

A massa de carbono encontrada para o Sistema 1 é próxima ao valor médio apresentado por Rocha (2011), que obteve massa de carbono na madeira correspondente a 8,63 t C ha ${ }^{-1}$ ano $^{-1}$, para um plantio de eucalipto com idade de 48 meses em espaçamento de $3 \times 3 \mathrm{~m}$.

Müller et al. (2009), estudando um Sistema silvipastoril misto, com E. grandis (Eucalipto) e Acacia mangium (Acácia), aos 10 anos, com densidade populacional de 105 árvores ha ${ }^{-1}$, sendo 60 de eucalipto e 45 de acácia, implantado na Zona da Mata Mineira, observaram massa de carbono de 1,43 t C.ha' ${ }^{-1}$ ano $^{-1}$.

Tsukamoto Filho et al. (2004), ao estudarem a fixação de carbono em um Sistema agrissilvipastoril com eucalipto, aos 11 anos, com espaçamento de 10 × 4 m, no município de Paracatu, MG, obtiveram uma massa de carbono na madeira correspondente a 5,17 t C ha-1 ano $^{-1}$.

Analisando os valores médios obtidos para a energia estocada por hectare ano $^{-1}$, verifica-se que, a exemplo da biomassa seca e massa de carbono, os valores obtidos também aumentaram à medida que a área útil por árvore foi reduzida. Isso pode ser explicado pelos maiores valores obtidos de biomassa seca no Sistema 1, além do maior poder calorífico superior da madeira para as árvores deste Sistema (Figura 1). 
Os resultados obtidos evidenciam a importância da produtividade volumétrica sobre as estimativas de biomassa seca, carbono e energia disponível nos Sistemas Agroflorestais avaliados. Vale destacar que no Sistema 1 a área útil por árvore é menor, ocasionando maior número de árvores por hectare e produção de maior volume de madeira, contribuindo para o incremento em biomassa seca e, consequentemente, incrementando os demais parâmetros avaliados.

No entanto, é necessário fazer uma análise técnica e financeira da produção de madeira destes Sistemas, considerando a competição entre as árvores com o tempo, os custos e as receitas advindas do desbaste para a produção de energia, a porcentagem de árvores retiradas e a idade deste desbaste.

\section{CONCLUSÕES}

Os Sistemas Agroflorestais avaliados apresentam potencial para a produção de biomassa seca de madeira para fins energéticos.

Os arranjos espaciais dos Sistemas Agroflorestais influenciaram na densidade básica e no poder calorífico da madeira de eucalipto.O teor de carbono não foi afetado pelo espaçamento.

O Sistema 1, em decorrência do menor espaçamento entre árvores, apresentou os maiores valores de biomassa seca de madeira, carbono e energia disponível.

\section{AGRADECIMENTOS}

Ao CNPq, CAPES e FAPEMIG, pela concessão de bolsas e financiamentos da pesquisa.

\section{REFERÊNCIAS BIBLIOGRÁFICAS}

ABNT - ASSOCIAÇÃO BRASILEIRA DE NORMAS TÉCNICAS - NBR 8633: Carvão vegetal - determinação do poder calorífico - método de ensaio. Brasília, 1984.

ABNT - ASSOCIAÇÃO BRASILEIRA DE NORMAS TÉCNICAS - NBR 11941: Madeira - determinação da densidade básica. Brasília, 2003.

ALVARES, C. A.; STAPE, J. L.; SENTELHAS, P. C.; GONÇALVES, J. L. M.; SPAROVEK, G. Köppen's climate classification map for Brazil. Meteorologische Zeitschrift, v. 22, n. 6, p. 711-728, 2013.

BERGER, R. Crescimento e qualidade da madeira de um clone de Eucalyptus saligna Smith sob o efeito do espaçamento e da fertilização, 2000. 110 p. Dissertação (Mestrado em Engenharia Florestal) - Universidade Federal de Santa Maria. Santa Maria, 2000.

BERGER, R.; SCHNEIDER P. R.; FINGER, C. A. G.; HASELEIN, C. R. Efeito do espaçamento e da adubação no crescimento de um clone de Eucalyptus saligna Smith. Ciência Florestal, Santa Maria, v. 12, n. 2, p. 75-87, 2002.

BRASIL. Plano setorial de mitigação e de adaptação às mudanças climáticas para a consolidação de uma economia de baixa emissão de carbono na agricultura: plano ABC (Agricultura de Baixa Emissão de Carbono). Brasília: MAPA/ACS, 2012. 173 p.

CORDEIRO, S. A.; SILVA, M. L. Análise técnica e econômica de Sistemas Agrossilvipastoris. In: OLIVEIRA NETO, N.; VALE, A. B.; NACIF, A. P.; VILAR, M. B.; ASSIS, J. B. (Org.). Sistema Agrossilvipastoril: integração lavoura, pecuária e floresta. Viçosa: SIF, 2010. p. 167-189.

DEBELL, D. S.; KEYES C. R.; GARTNER, B. L. Wood density of Eucalyptus saligna grown in Hawaiian plantations: effects of silvicultural practices and relation to growth rate. Australian Forestry, Melbourne, v. 64, n. 2, p. 106-110, 2001. 
Torres et al. - Estimativas da produção e propriedades da madeira de eucalipto em Sistemas Agroflorestais

ELLIOTT, G. K. Wood density in conifers. Oxford: Commonwealth Agricultural Bureaux, 1970.44 p. (Comunicação Técnica, 8)

FERREIRA, M.; KAGEYAMA, P. Y. Melhoramento genético da densidade da madeira de eucalipto. Boletim Informativo IPEF, Piracicaba, v. 6, n. 20, p. A1-A15, 1978.

FONTAN, I. C. I.; REIS, G. G.; REIS, M. G. F.; LEITE, H. G.; MONTE, M. A.; RAMOS, D. C.; SOUZA, F. C. Growth of pruned eucalypt clone in an agroforestry system in southeastern Brazil. Agroforestry Systems, Dordrecht, v. 83, n. 2, p. 121-131, 2011.

FRANKE, I. L.; FURTADO, S. C. Sistemas silvipastoris: fundamentos e aplicabilidade. Rio Branco: Embrapa Acre, 2001. $51 \mathrm{p}$ (Documentos, 74).

GONÇALVES, J. L. M.; STAPE, J. L.; LACLAU, J.-P.; SMETHURST, P.; GAVA, J. L. Silvicultural effects on the productivity and wood quality of eucalypt plantations. Forest Ecology and Management, Amsterdam, v. 193, n. 1-2, p. 45-61, 2004.

HASELEIN, C. R.; BERGER, R.; GOULART, M. Propriedades de flexão estática da madeira e umidade e a $12 \%$ de umidade de um clone de Eucalyptus saligna Smith sob efeito do espaçamento e da adubação. Ciência Florestal, Santa Maria, v. 12, n. 2, p. 147-152, 2002.

LEITE, H. G.; NOGUEIRA, G. S.; MOREIRA, A. M. Efeito do espaçamento e da idade sobre variáveis de povoamentos de Pinus taeda L. Revista Árvore, v. 30, n. 4, p. 603-612, 2006.

MANFREDI, V.; BARICHELO, L. E. G. Variação do rendimento de celulose sulfato ao longo do tronco do eucalipto. In: CONGRESSO ANUAL DA ABCP, 18, 1985, São Paulo. Anais... São Paulo: ABCP. 1985. p. 5-29.

MÜller, M. D.; FERnANDES, E. N.; CASTRO, C. R. T.; PACIUllo, D. S. C.; ALVES, F. F. Estimativa de acúmulo de biomassa e carbono em Sistema Agrossilvipastoril na Zona da Mata Mineira. Pesquisa Florestal Brasileira, Colombo, n. 60, p. 11-17, 2009.

OliveirA, A. C.; CARneiro, A. C. O.; VITAL, B. R.; AlMEIDA, W.; PEREIRA, B. L. C.; CARDOSO, M. T. Parâmetros de qualidade da madeira e do carvão vegetal de Eucalyptus pellita F. Muell. Scientia Forestalis, Piracicaba, v. 38, n. 87, p. 431-439, 2010.

OLIVEIRA, J. T. S.; HELLMEISTER, J. C.; SIMÕES, J. W.; TOMAZELLO FILHO, M. Caracterização da madeira de sete espécies de eucaliptos para a construção civil: 1 - avaliações dendrométricas das árvores. Scientia Forestalis, Piracicaba, n. 56, p. 113-124, 1999.

OLIVEIRA NETO, S. N.; PAIVA, H. N. Implantação e manejo do componente arbóreo em Sistema Agrossilvipastoril. In: OLIVEIRA NETO, N.; VALE, A. B.; NACIF, A. P.; VILAR, M. B.; ASSIS, J. B. (Org.). Sistema Agrossilvipastoril: integração lavoura, pecuária e floresta. Viçosa: SIF, 2010. p. 15-68.

OLIVEIRA NETO, S. N.; REIS, G. G.; REIS, M. G. F. Eucalipto: as questões ambientais e seu potencial para Sistemas agrossilvipastoris. In: FERNANDES, E. N.; PACUILLO, D. S.; CASTRO, C. R. T.; MÜLLER, M. D.; ARCURI, P. B.; CARNEIRO, J. C. (Ed.) Sistemas agrossilvipastoris na América do Sul: desafios e potencialidades. Juiz de Fora: Embrapa Gado de Leite, 2007. p. 245-282.

OLIVEIRA NETO, S. N.; REIS, G. G.; REIS, M. G. F.; NEVES, J. C. L. Produção e distribuição de biomassa em Eucalyptus camaldulensis Dehn. em resposta à adubação e ao espaçamento. Revista Árvore, Viçosa, v. 27, n. 1, p. 15-23, 2003. 
PAULA, R. R.; REIS, G. G.; REIS, M. G. F.; OLIVEIRA NETO, S. N.; LEITE H. G.; MELIDO, R. C. N.; LOPES, H. N. S.; SOUZA, F. C. Eucalypt growth in monoculture and silvopastoral systems with varied tree initial densities and spatial arrangements. Agroforestry Systems, Dordrecht, v. 87, n. 6, p. 1295-1307, 2013.

PEREIRA, B. L. C.; CARNEIRO, A. C. O.; CARVAlHO, A. M. M. L.; COlODETTE, J. L.; OliVEIRA, A. C.; FONTES, M. P. F. Influence of Chemical Composition of Eucalyptus Wood on Gravimetric Yield and Charcoal Properties. BioResources, Raleigh, v. 8, n. 3, p. 4574-4592, 2013.

PRASAD, J. V. N. S.; KORWAR, G. R.; RAO, K. V.; MANDA, U. K.; RAO, C. A. R.; RAO, G. R.; RAMAKRISHMA, Y. S.; VENKATESWARLU, B.; RAO, S. N.; KULMARNI, H. D.; RAO, M. R. Tree row spacing affected agronomic and economic performance of Eucalyptus-based agroforestry in Andhra Pradesh, Southern India. Agroforestry Systems, Dordrecht, v. 78, n. 3, p. 253-267, 2010.

PROTÁSIO, T. P.; GOULART, S. L.; NEVES, T. A.; ASSIS, M. R.; TRUGILHO, P. F. Clones comerciais de Eucalyptus de diferentes idades para o uso bioenergético da madeira. Scientia Forestalis, Piracicaba, v. 42, n. 101, p. 113-127, 2014.

RAMOS, A. M.; SANTOS, L. A. R. D.; FORTES, L. T. G. Normais climatológicas do Brasil 1961-1990: edição revista e ampliada. Brasília: INMET, 2009.

ROCHA, M. F. V. Influência do espaçamento e da idade na produtividade e propriedades da madeira de Eucalyptus grandis x Eucalyptus camaldulensis para energia, 2011. 71 p. Dissertação (Mestrado em Ciência Florestal) - Universidade Federal de Viçosa. Viçosa, 2011.

SANTANA, W. M. S. Crescimento, produção e propriedades da madeira de um clone de Eucalyptus grandis e E. urophylla com enfoque energético, 2009. 92 p. Dissertação (Mestrado em Processamento e utilização de madeira) - Universidade Federal de Lavras. Lavras, 2009.

SANTOS, L. C.; CARVAlHO, A. M. M. L.; PEREIRA, B. L. C.; OlIVEIRA, A. C.; CARNEIRO, A. C. O.; TRUGILHO, P. F. Propriedades da madeira e estimativas de massa, carbono e energia de clones de Eucalyptus plantados em diferentes locais. Revista Árvore, Viçosa, v. 36, n. 5, p. 971-980, 2012.

SANTOS, M. D. Efeito do espaçamento de plantio na biomassa do fuste de um clone híbrido interespecífico de Eucalyptus grandis e Eucalyptus urophylla. 2011. 140 p. Dissertação (Mestrado em Ciência Florestal) Universidade Estadual Paulista. Botucatu, 2011.

SANTOS, R. C.; CARNEIRO, A. C. O.; CASTRO, A. F. M.; CASTRO, R. V. O.; BIANCHE, J. J.; SOUZA, M. M.; CARDOSO, M. T. Correlações entre os parâmetros de qualidade da madeira e do carvão vegetal de clones de eucalipto. Scientia Forestalis, Piracicaba, v. 39, n. 90, p. 221-230, 2011.

SOUZA, A. P.; GASPAR, M.; TINÉ, M. A. S.; BUCKERIDGE, M. S. Ajustando os botões: como as plantas lidam com o aumento do $\mathrm{CO}_{2}$ atmosférico. In: BUCKERIDGE, M. S. (Org.). Biologia \& Mudanças Climáticas no Brasil. São Carlos: Rima Editora. p. 101-113, 2008.

TREVISAN, R.; DENARDI, L.; HASELEIN, C. R.; GATTO, D. A. Efeito do desbaste e variação longitudinal da massa específica básica da madeira de Eucalyptus grandis. Scientia Forestalis, Piracicaba, v. 40, n. 95, p. 393$399,2012 b$

TREVISAN, R.; ELOY, E.; DENARDI, L.; HASELEIN, C. R.; CARON, B. O. Variação axial e efeito do desbaste na massa específica das árvores centrais de Eucalyptus grandis. Ciência Rural, Santa Maria, v. 42, n. 2, p. 312-318, 2012a. 
Torres et al. - Estimativas da produção e propriedades da madeira de eucalipto em Sistemas Agroflorestais

TRUGILHO, P. F.; ARANTES, M. D. C.; PÁDUA, F. A.; ALMADO, R. P.; BALIZA, A. E. R. Estimativa de carbono fixado na madeira de um clone híbrido de Eucalyptus urophylla e Eucalyptus grandis. Cerne, Lavras, v. 16, n. suplemento, p. 33-40, 2010.

TSUKAMOTO FILHO, A. A.; COUTO, L.; NEVES, J. C. L.; PASSOS, C. A. M.; SILVA, M. L. Fixação de carbono em um Sistema agrissilvipastoril com eucalipto na região do cerrado de Minas Gerais. Agrossilvicultura, v. 1, n. 1, p. 29-41, 2004.

VITAL, B. R. Métodos de determinação da densidade da madeira. Viçosa: SIF, 1984. 21 p.

WASHUSEN, R.; BAKER, T.; MENZ, D.; MORROW, A. Effect of thinning and fertilizer on the cellulose crystallite width of Eucalyptus globulus. Wood Science and Technology, New York, v. 39, n. 7, p. 569-578, 2005.

ZOBEL, B. The changing quality of the world wood supply. Wood Science and Technology, New York, v. 18, n. 1 , p. $1-17,1984$.

Recebido em 09/10/2014

Aceito para publicação em 12/08/2015 